\title{
An Uncommon Potentially Fatal Complication in a Patient without Predisposing Factor Following Oral Bowel Preparation Commonly Used for Colonoscopy
}

\author{
Iris Wai Sum Li ${ }^{*}$, Ling Pong Leung², Sik Hon Tsui ${ }^{3}$ \\ ${ }^{1}$ Queen Mary Hospital, Hospital Authority, Hong Kong, China \\ ${ }^{2}$ Emergency Medicine Unit, Li Ka Shing Faculty of Medicine, The University of Hong Kong, Hong Kong, China \\ ${ }^{3}$ Department of Accident \& Emergency, Queen Mary Hospital, Hospital Authority, Hong Kong Special \\ Administrative Region, Hong Kong, China \\ Email: *irisli.irisli.irisli@gmail.com
}

Received 1 January 2016; accepted 18 March 2016; published 21 March 2016

Copyright (C 2016 by authors and Scientific Research Publishing Inc.

This work is licensed under the Creative Commons Attribution International License (CC BY). http://creativecommons.org/licenses/by/4.0/

(c) (i) Open Access

\begin{abstract}
We described the case report of a patient presented with no known predisposing factor, generalized malaise with vomiting, locked jaw, perioral paraesthesia, carpopedal spasm and a positive Chvostek's sign which resulted from severe electrolyte disturbance at 5 hours after use of oral sodium phosphate solution (NaPO4) as bowel preparation for colonoscopy. On presentation, she developed hyperphosphatemia and symptomatic hypocalcaemia with serum phosphate of 1.84 mmol/L and adjusted calcium level $1.67 \mathrm{mmol} / \mathrm{L}$ respectively. Her symptoms subsided after immediate intravenous calcium gluconate followed by oral calcium supplement. The electrolyte disturbances were normalized with an uneventful clinical course. The potentially fatal complications of sodium phosphate solution which commonly prescribed as bowel preparation for conventional colonoscopy could develop though previously reported as uncommon in patients without predisposing factors, and should not be overlooked. Urgent assessment and immediate correction of electrolyte disturbances are needed. Recommendations on patient selection of use of sodium phosphate to minimize risk of developing adverse events are needed to incorporate in clinical protocols.
\end{abstract}

\section{Keywords}

Symptomatic Hypocalcaemia, Hyperphosphatemia, Sodium Phosphate Solution, Bowel

\footnotetext{
*Corresponding author.
} 


\section{Preparation, Colonoscopy}

\section{Introduction}

Oral sodium phosphate solution (NaPO4) has been prescribed commonly in clinical practice for bowel preparation before conventional colonoscopy and is generally considered as commonly tolerated without adverse reaction except in high-risk patients. A healthy woman developed symptomatic hypocalcaemia and hypophosphatemia presented with paraesthesia, carpopedal spasm and positive Chvostek's sign at 5 hours after oral NaPO4 for colonoscopy, with an uneventful course after immediate intravenous and oral calcium preparation. We should be aware of potentially-fatal complications from the commonly prescribed NaPO4 can occur even in normal person.

\section{Case Report}

A 60 year old lady who was a hepatitis B virus carrier without evidence of liver cirrhosis, and not on anti-viral and other medications, attended emergency department on one mid-night of July 2015 for generalized malaise with vomiting, locked jaw, perioral paraesthesia, carpopedal spasm and a positive Chvostek's sign at approximately 5 hours and 3 hours after the first and second doses of oral sodium phosphate solution $(45 \mathrm{ml}$ each dose of CB Fleet Co Inc, Lynchburg, VA, USA) respectively as bowel preparation regimen for conventional colonoscopy for body check-up to be performed the next morning. She had bowel opening for 7 times with nonbloody loose stool before presentation. At the time of presentation, she was hemodynamically stable but clinically dehydrated. Her serum ionized calcium and bicarbonate levels were $0.82 \mathrm{mmol} / \mathrm{L}$ and $23.3 \mathrm{mmol} / \mathrm{L}$ respectively, by i-STAT handheld blood analyzer. The serum level of corrected total calcium was $1.67 \mathrm{mmol} / \mathrm{L}$ (normal range 2.24 to $2.63 \mathrm{mmol} / \mathrm{L}$ ), phosphate $1.84 \mathrm{mmol} / \mathrm{L}$ (normal range 0.88 to $1.45 \mathrm{mmol} / \mathrm{L}$ ), albumin 41 (normal range 39 to $50 \mathrm{~g} / \mathrm{L}$ ), urea $3.4 \mathrm{mmol} / \mathrm{L}$ (normal range 2.9 to $8.0 \mathrm{mmol} / \mathrm{L}$ ) and creatinine $81 \mu \mathrm{mol} / \mathrm{L}$ (normal range 49 to $82 \mu \mathrm{mol} / \mathrm{L}$ ). The electrocardiography showed normal sinus rhythm with a rate of 67 per minute, the QT interval was $407 \mathrm{~ms}$. Fluid replacement was given, and her symptoms subsided after treatment with intravenous calcium gluconate ( $40 \mathrm{ml} 10 \%, 9.0 \mathrm{mmol}$ ) followed by oral calcium carbonate chewable tablets $1000 \mathrm{mg}$ twice daily. Her serum adjusted calcium was $1.97 \mathrm{mmol} / \mathrm{L}$ at 6 hours after admission while on calcium replacement, and was $2.33 \mathrm{mmol} / \mathrm{L}$ and with normal renal function at 3 weeks after discharge without any calcium supplements (Figure 1). The colonoscopy intended for body-check up had been cancelled.

\section{Discussion}

This is the report of symptomatic hypocalcaemia and hyperphosphatemia in a patient with normal renal function and serum albumin level without other known predisposing factors for electrolyte disturbances after use of oral sodium phosphate solution as bowel preparation for conventional colonoscopy. Severe electrolyte disturbances resulted from sodium phosphate bowel preparation leading to severe symptoms and complications have been described in patients with known predisposing factors. The incidence of developing hyperphosphatemia and hypocalcaemia in patients without contraindication to sodium phosphates were reported as $39 \%$ and $5 \%$ respectively in a prospective randomized blinded study. It was also reported that $80 \%$ of patients using oral sodium phosphates solution had a $\geq 5 \%$ increase in phosphate levels of baseline, $\geq 65 \%$ patients had a $\geq 10 \%$ increase, and $\geq 25 \%$ patients had a $\geq 50 \%$ increase respectively [1]. Our patient had a lower serum adjusted calcium level, and higher phosphate level on presentation as compared to the mean levels of calcium $(2.3 \mathrm{mmol} / \mathrm{L})$ and phosphates $(1.4 \mathrm{mmol} / \mathrm{L})$ after intake of sodium phosphates solution in this study [1].

The mechanism of electrolyte disturbances resulted from NaPO4 could be explained by its nature and mechanisms of action for bowel preparation. Oral NaPO4 is a saline mixture which acts by osmotic processes to increase fluid in the small intestinal lumen which produces bowel distension and leads to peristalsis and colonic evacuation. In general, the responses usually occur at 30 minutes after intake but may take as long as 6 hours and are subjected to individual variations. The high sodium and phosphate content of oral NaPO4 render them as osmotic purgatives. Each $45 \mathrm{ml}$ dose of oral $\mathrm{NaPO} 4$ has a sodium content of $5 \mathrm{~g}$, and each ml contains $4.82 \mathrm{mmol}$ sodium and $12.45 \mathrm{mmol}$ phosphate, and supplied in a solution of $748 \mathrm{mOsm} / 45 \mathrm{ml}$. Therefore, dehydration, hyperphosphatemia, hypocalcaemia, hypernatraemia, other electrolyte abnormalities and associated complications 


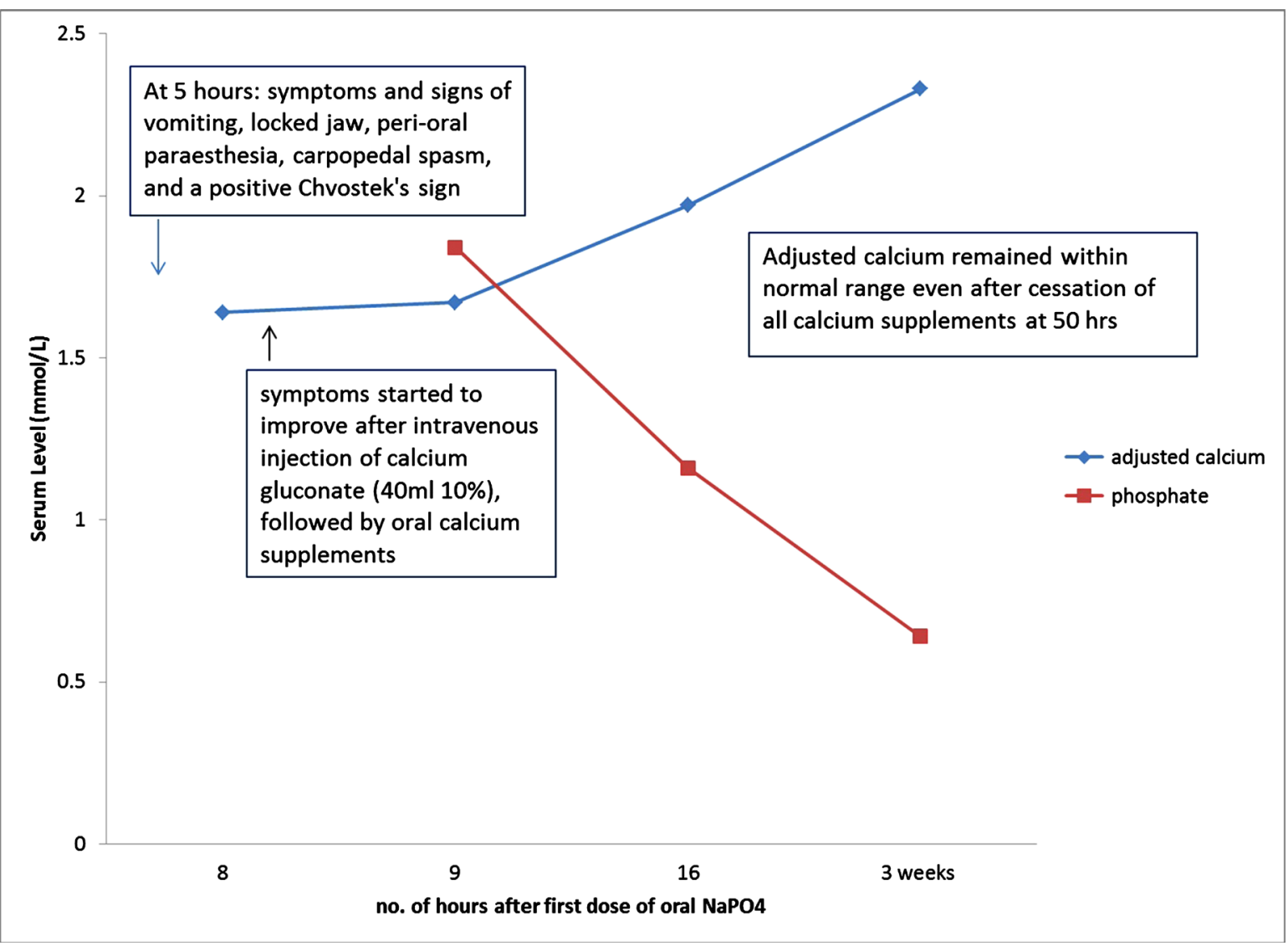

Figure 1. Illustration showing the evolution of the patient's symptoms and signs, and serum levels of adjusted calcium and phosphate.

may occur as adverse reaction of oral NaPO4 [2] [3].

Despite the fact that a higher proportion of patients using sodium phosphate developed electrolyte disturbances as compared to those using polyethylene glycol-electrolyte solution (PEG-ELS), and there was no significant differences in the clinical efficacy between sodium phosphate and various preparation of PEG in achieving an adequate bowel cleansing, use of two doses of sodium phosphate solution had been shown to achieve a better bowel preparation than PEG, and are commonly used in clinical practice nowadays than PEG-ELS or sulphate-free (-SF) solution for bowel preparation for conventional colonoscopy of diagnostic and therapeutic purposes [1] [4] [5]. It is mainly due to better patient tolerability with less volume of sodium phosphates required, though intake of at least 2 liters of other clear fluid is still needed, as compared to the intake of 2 to 4 liters of osmotically balanced non-absorbable solution of PEG with or without salty taste; and more dizziness and anal irritation had been noted in patients receiving 2 than 1 dose of sodium phosphates solution [1] [4] [5].

Our patient did not have any predisposing factors for hyperphosphatemia and hypocalcaemia after use of sodium phosphate. She had bowel opening after use of sodium phosphate and before symptom onset, this made the diminished intestinal motility as contributing factor less likely. Predisposing factors for phosphate accumulation in serum include decrease in urinary phosphate excretion or excessive intestinal absorption of phosphate or inadequate parathyroid hormone response or in combination. Also aged $>65$, renal impairment with or without renal replacement therapy, congestive heart failure, liver cirrhosis, use of angiotensin-converting enzyme inhibitors or angiotensin II receptor antagonists or diuretics, intestinal obstruction, inflammatory disorders, and hypoparathyroidism as a result of surgery or autoimmune diseases or resistance can lead to hyperphosphatemia. The hyperphosphatemia then also induces hypocalcaemia.

Other electrolyte disturbances that commonly reported after use of sodium phosphate solution include significant increase or decrease in serum sodium, decrease in serum potassium, magnesium, bicarbonate and impaired renal function. These were not observed in our patient [1] [4] [6]. 
The spectrum of complications of sodium phosphate bowel preparation mainly due to hyperphosphatemia and hypocalcaemia can be potentiated by clinical adverse effects from coexisting of other electrolyte disturbances. It ranges from mild dehydration, hypovolemia, asymptomatic electrolyte disturbances, to tetany, mental confusion, arrhythmia, renal impairment and death [1]. Our patient developed locked jaw, carpopedal spasm and positive Chvostek's sign at approximately 5 hours after the first dose of sodium phosphate solution at a clinical time course in concordance with previous reports [2] [7]. The short interval of 2 hours between the first and second doses of oral sodium phosphate solution taken on the same evening led to rapid accumulation of intestinal phosphate level and subsequent systemic absorption was suspected to be the cause resulted in hyperphosphatemia and symptomatic hypocalcaemia in our patient. No subsequent morbidity and mortality developed in our patient. Acute kidney injury and chronic renal impairment after use of sodium phosphate solution was not observed in our patient as reflected by the normal renal function during hospitalization and on follow up respectively. The incidence of acute kidney injury after use of sodium phosphate, which is also known as acute phosphate nephropathy, was reported in a range of $1 \%$ to $4 \%$, which is similar to that of contrast nephropathy in general population [7]. Despite the mechanism of kidney injury from use of sodium phosphate solution is multifactorial including those predisposing factors described above, renal biopsy of these patients uniformly showed deposition of calcium-phosphate crystals as nephrocalcinosis leading to tubulointerstitial nephropathy [2] [6] [8]. The trigger of innate immune system with release of pro-inflammatory mediators in response to intraluminal calcium-phosphate crystal deposition and binding to tubular epithelial cells leading to chronic inflammation and fibrosis have also been hypothesized as possible mechanism of kidney injury after use of sodium phosphate [2] [8]. On the other hand, the complication from use of sodium phosphate may also potentiate acute renal failure in those with concomitant use of nephrotoxic medications such as non-steroidal anti-inflammatory drugs, and metformin which is associated with metformin-associated lactic acidosis [9]. The mortality resulted from using sodium phosphate was reported as high as $33 \%$ in patients with impaired renal function, high serum phosphate levels or co-morbidities. Majority of these mortality occurred in patients at extremities of age [8].

Seemingly safe and commonly prescribed bowel-cleansing agents in clinical practice can cause severe electrolyte disturbances which are potentially fatal. Symptomatic hypocalcaemia and serum adjusted calcium level $<1.9 \mathrm{mmol} / \mathrm{L}$ should be urgently assessed for admission, investigation and treatment [10]. Recommendations to minimize the renal risks and other adverse events with use of oral sodium phosphate have been reported. These include appropriate patient selection, adequate hydration and proper dosing. Oral sodium phosphate is not recommended in patients with renal failure, congestive heart failure, significant ischemia heart disease, ascites, ileus or bowel obstruction, pregnancy, age younger than 18 , incapable to follow instructions or ensure adequate hydration, use of medications include diuretics and angiotensin-converting enzyme/angiotensin II receptor antagonists. Alternative bowel cleansing agent such as PEG-ELS or SF solutions will need to be considered in these cases. Before colonoscopy is performed, adequate hydration is recommended by encouraging oral intake of up to 2 to 3 liters fluid, minimizing phosphate load and intravascular volume shift by widening the dosing interval to 10 to 12 hours apart, and the second dose is recommended to be taken in the morning of the procedure; on the contrary, our patient had both doses taken on the same evening prior to the procedure. During colonoscopy, intravenous saline is recommended to maintain adequate hydration. After colonoscopy, oral fluid intake is encouraged and blood for renal function to look for electrolyte or renal function abnormalities is recommended, and should be urgently treated accordingly if any with subsequent close monitoring [7] [11].

\section{Conclusion}

In summary, oral sodium phosphate solution for bowel preparation is commonly used in clinical practice as bowel preparation for colonoscopy because of better patient tolerability. Its adverse effects resulted from hyperphosphatemia and hypocalcaemia can develop even in patients without predisposing factors and should not be overlooked, though severe complications and mortality are usually reported in patients with predisposing factors. Symptomatic or asymptomatic electrolyte disturbances should be urgently treated. Careful patient selections together with precautionary measures are needed to minimize the development of adverse effects from use of oral sodium phosphate.

\section{Conflict of Interest}

All authors have no conflict of interest. 


\section{References}

[1] Mathus-Vliegen, E.M. and Kemble, U.M. (2006) A Prospective Randomized Blinded Comparison of Sodium Phosphate and Polyethylene Glycol-Electrolyte Solution for Safe Bowel Cleansing. Alimentary Pharmacology \& Therapeutics, 4, 543-552. http://dx.doi.org/10.1111/j.1365-2036.2006.02777.x

[2] Heher, E.C., Thier, S.O., Rennke, H. and Humphreys, B.D. (2008) Adverse Renal and Metabolic Effects Associated with Oral Sodium Phosphate Bowel Preparation. Clinical Journal of the American Society of Nephrology, 5, 1494-503. http://dx.doi.org/10.2215/CJN.02040408

[3] Product Information Fleet ${ }^{\circledR}$ Phospho-Soda ${ }^{\circledR}(2012)$ URL. https://gp2u.com.au/static/pdf/F/FLEET_PHOSPHO-SODA-PI.pdf

[4] Belsey, J., Epstein, O. and Heresbach, D. (2007) Systemic Review: Oral Bowel Preparation for Colonoscopy. Alimentary Pharmacology \& Therapeutics, 4, 373-384.

[5] Law, W.L., Choi, H.K., Chu, K.W., Ho, J.W. and Wong, L. (2004) Bowel Preparation for Colonoscopy: A Randomized Controlled Trial Comparing Polyethylene Glycol Solution, One Dose and Two Doses of Oral Sodium Phosphate Solution. Asian Journal of Surgery, 2, 120-124. http://dx.doi.org/10.1016/S1015-9584(09)60324-9

[6] Mok, M.M., Yip, T., Lui, S.L., Chan, D.T., Lai, K.N. and Lo, W.K. (2011) Severe Hypocalcaemia and Hyperphosphataemia Caused by Oral Sodium Phosphate Fleet Solution in a Haemodialysis Patient after Parathyroidectomy. NDT Plus, 4, 248-250. http://dx.doi.org/10.1093/ndtplus/sfr070

[7] Lien, Y.H. (2008) Is Bowel Preparation before Colonoscopy a Risky Business for the Kidney? Nature Clinical Practice Nephrology, 4, 606-614. http://dx.doi.org/10.1038/ncpneph0939

[8] Woo, Y.M., Crail, S., Curry, G. and Geddes, C.C. (2006) A Life Threatening Complication after Ingestion of Sodium Phosphate Bowel Preparation. BMJ, 333, 589-590. http://dx.doi.org/10.1136/bmj.333.7568.589

[9] Hsu, W.H., Lin, C.H., Tai, Y.K. and Chao, T.B. (2012) Lactic Acidosis and Acute Renal Failure after Oral Sodium Phosphate for Bowel Preparation in a Diabetic Patient Using Metformin-A Case Report. The Journal of Society of Colon and Rectal Surgeon (Taiwan), 23, 72-75.

[10] Cooper, M.S. and Gittoes, N.J.L. (2008) Diagnosis and Management of Hypocalaemia. BMJ, 336, 1298-1302. http://dx.doi.org/10.1136/bmj.39582.589433.BE

[11] Rex, D.K. and Vanner, S.J. (2009) Colon Cleansing before Colonoscopy: Does Oral Sodium Phosphate Solution Still Make Sense? Canadian Journal of Gastroenterology, 23, 210-214. 